\title{
Input-Specific Immunolocalization of Differentially Phosphorylated Kv4.2 in the Mouse Brain
}

\author{
Andrew W. Varga, ${ }^{1}$ Anne E. Anderson, ${ }^{1,2}$ J. Paige Adams, ${ }^{1}$ Hannes Vogel, ${ }^{3}$ and \\ J. David Sweatt ${ }^{1,4}$ \\ ${ }^{1}$ Division of Neuroscience, ${ }^{2}$ Departments of Pediatrics and Neurology, ${ }^{3}$ Department of Pathology, Baylor College of Medicine, \\ Houston, Texas 77030, USA
}

\begin{abstract}
Voltage-gated A-type potassium channels such as Kv4.2 regulate generation of action potentials and are localized abundantly in the hippocampus and striatum. Phosphorylation consensus sites for various kinases exist within the sequence of the potassium channel subunit Kv4.2, including consensus sites for extracellular signal-regulated kinase/mitogen activated protein kinase (ERK/MAPK), protein kinase A (PKA), protein kinase $\mathrm{C}$ (PKC), and calcium/calmodulin-dependent kinase II (CaMKII), and kinase assays have shown that particular amino acids of the consensus sites are bonafide phosphorylation sites in vitro. We have developed antibodies recognizing Kv4.2 triply phosphorylated at the three ERK sites as well as two antibodies recognizing singly phosphorylated Kv4.2 channels at the PKA sites (one amino-terminal and one carboxy-terminal). In the present study, we report the development of reliable immunohistochemistry protocols to study the localization of these phosphorylated versions of $\mathrm{Kv} 4.2$, as well as total $\mathrm{Kv} 4.2$ in the mouse brain. A general description of the areas highlighted by these antibodies includes the hippocampus, amygdala, cortex, and cerebellum. Such areas display robust synaptic plasticity and have been implicated in spatial, associative, and motor learning. Interestingly, in the hippocampus, the antibodies to differentially phosphorylated Kv 4.2 channels localize to specific afferent pathways, indicating that the Kv4.2 phosphorylation state may be input specific. For example, the stratum lacunosum moleculare, which receives inputs from the entorhinal cortex via the perforant pathway, displays relatively little ERK-phosphorylated Kv4.2 or PKA carboxy-terminal-phosphorylated Kv4.2. However, this same layer is highlighted by antibodies that recognize Kv4.2 that has been phosphorylated by PKA at the amino terminus. Similarly, of the three antibodies tested, the soma of CA3 neurons are primarily recognized by the ERK triply phosphorylated Kv4.2 antibody, and the mossy fiber inputs to CA3 are primarily recognized by the carboxy-terminal PKA-phosphorylated Kv4.2. This differential phosphorylation is particularly interesting in two contexts. First, phosphorylation may be serving as a mechanism for targeting. For example, the amino-terminal PKA phosphorylation may be acting as a tag for a discrete pool of Kv4.2 to enter stratum lacunosum moleculare. Second, as phosphorylation may regulate channel biophysical properties, differential phosphorylation of Kv4.2 in the dendrites of pyramidal neurons may confer unique biophysical properties upon particular dendritic input layers.
\end{abstract}

Much interest has been generated recently in the potassium channel subunit Kv4.2 for its critical role in regulating membrane excitability. Several lines of evidence suggest that Kv4.2 underlies the A-type current $\left(\mathrm{I}_{\mathrm{A}}\right)$ in neurons (Serodio et al. 1996; Song et al. 1998) as well as the transient outward current $\left(\mathrm{I}_{\mathrm{to}}\right)$ in cardiac ventricular myocytes (Barry et al. 1998). In particular, Tkatch, et. al. (2000) have found that Kv4.2 mRNA abundance and A-type current amplitude are linearly related in the striatum. In the hippocampus, such A-type current has been found to dampen back-propagation of action potentials in the distal dendrites of CA1 pyramidal neurons, thereby setting up the capacity to modify the EPSP or back-propagating action potentials following synaptic activity (Hoffman et al. 1997). Immunohistochemical studies

${ }^{4}$ Corresponding author.

E-MAIL jsweatt@bcm.tmc.edu; FAX (713) 798-3946.

Article and publication are at www.learnmem.org/cgi/doi/ $10.1101 / \mathrm{lm} .35300$. show that Kv4.2 protein localizes abundantly to the hippocampus (Sheng et al. 1992) with particular localization to the neuronal soma and dendrites (Maletic-Savatic et al. 1995), structures in which modulating membrane excitability could have important implications for LTP and memory. Furthermore, ultrastructural studies in supraoptic neurons have shown that $\mathrm{Kv} 4.2$ is localized to the postsynaptic membrane directly across from the presynaptic terminal (Alonso and Widmer 1997).

Consensus sites for various kinases exist within the Kv4.2 amino acid sequence including consensus sites for protein kinase A (PKA), protein kinase (PKC), extracellularsignal regulated kinase (ERK), and calcium/calmodulin-dependent kinase II (CaMKII). We have shown that $\mathrm{Kv} 4.2$ is a substrate for both ERK and PKA in vitro as well as in hippocampal area CA1 ( Anderson et al. 2000; Adams et al. 2000). Antibodies recognizing a triply phosphorylated Kv4.2 at the ERK sites (Adams et al. 2000) as well as two

LEARNING \& MEMORY 7:321-332 @ 2000 by Cold Spring Harbor Laboratory Press ISSN1072-0502/00 \$5.00

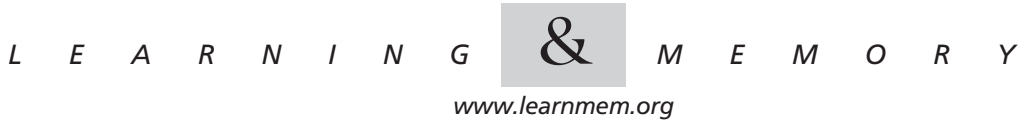


singly phosphorylated Kv4.2 channels at the PKA sites (one amino-terminal and one carboxy-terminal) (Anderson et al. 2000) have been developed. PKA and ERK are particularly interesting as modulators of $\mathrm{Kv} 4.2$ due to their known role in LTP induction (English and Sweatt 1996; Roberson et al. 1999; Wong et al. 1999) and learning and memory (Atkins et al. 1998; Impey et al. 1998a; Blum et al. 1999; Selcher et al. 1999; Wong et al. 1999). In this context, it is interesting that PKA activation shifts the activation curve for A-type channels $\sim 15 \mathrm{mV}$ in the depolarizing direction in the distal dendrites of CA1 pyramidal neurons (Hoffman and Johnston 1998). Thus, if Kv4.2 influences membrane excitability, then it is likely that modulation of Kv4.2 through phosphorylation events could also affect excitability and the probability of inducing depolarization-dependent synaptic plasticity.

Given these considerations, our understanding of hippocampal synaptic plasticity would be well served by the ability to identify the distribution of $\mathrm{Kv} 4.2$ in its different phosphorylated states. In the present study, we utilized Kv4.2 phospho-site-specific antibodies in immunohistochemical studies with the aim of localizing the phosphorylated isoforms of Kv4.2. Specifically, we sought to determine whether there existed a differential distribution of phosphorylated isoforms of Kv4.2 in the hippocampus and whether such patterns might elucidate potential mechanisms by which Kv4.2 contributes to regulating the function of hippocampal circuitry. In addition to the hippocampus, we looked at the allocation of phosphorylated isoforms of $\mathrm{Kv} 4.2$ in other brain areas prominently involved in memory formation such as the cerebellum, amygdala, and neocortex.

Differential distribution of Kv4.2 phospho-isoforms was most obvious in the hippocampus in which three areas displayed antibody specificity. These areas included (1) the stratum lacunosum moleculare, which was primarily recognized by the amino-terminal PKA-phosphorylated Kv4.2 antibody of the three antibodies tested, (2) the mossy fiber inputs into area CA3 that were primarily recognized by the carboxy-terminal PKA-phosphorylated Kv4.2, and (3) the CA3 soma that were primarily recognized by the ERK triply phosphorylated Kv4.2. Such input specificity may be the result of Kv4.2 phospho-isoform-specific targeting. Furthermore, because phosphorylation may regulate the biophysical properties of $\mathrm{Kv} 4.2$, an intriguing possibility is that phosphorylation of Kv4.2 may be a means for differentially summating discrete electrical inputs onto populations of neurons.

\section{RESULTS}

\section{Antibody Description and Specificity}

Prior observations indicating Kv4.2's ability to control membrane excitability, along with its colocalization with a variety of kinases in the hippocampus suggest to us that it might be a likely candidate for kinase regulation. Consistent with this idea, a scan of the Kv4.2 amino acid sequence manifests ERK consensus sequences in the Kv4.2 carboxyl terminus and PKA consensus sequences in both the amino- and carboxy-termini. Moreover, prior in vitro phosphorylation assays revealed that the $\mathrm{Kv} 4.2$ carboxyl terminus is phosphorylated in vitro by both ERK and PKA, whereas the amino terminus is phosphorylated by PKA but not ERK (Fig. 1). Direct amino acid sequencing revealed the ERK phosphorylation sites to be $\mathrm{Thr}^{602}$, $\mathrm{Thr}^{607}$, and $\mathrm{Ser}^{616}$, and the PKA phosphorylation sites to be $\mathrm{Thr}^{38}$ and $\mathrm{Ser}^{552}$ (Anderson et al. 2000; Adams et al. 2000).

The generation of antibodies recognizing specific Kv4.2 phospho-isoforms would be useful for determining the physiological significance of phosphorylation as well as for monitoring any changes that occur in the Kv4.2 phosphorylation state. With this in mind, we created three different phospho-specific antibodies to Kv4.2. The ERK triply phosphorylated antibody was made against a peptide running from AA586 to AA618 in which the ERK phosphorylation sites $\mathrm{Thr}^{602}$, $\mathrm{Thr}^{607}$, and $\mathrm{Ser}^{616}$ are phosphorylated (Adams et al. 2000). The carboxy-terminal PKA-phosphorylated Kv4.2 antibody was made against a peptide running from AA546 to AA558, in which the PKA phosphorylation site at $\mathrm{Ser}^{552}$ is phosphorylated. The amino-terminal PKAphosphorylated Kv4.2 antibody was made against a peptide running from AA32 to AA44, in which the PKA phosphorylation site at $\mathrm{Thr}^{38}$ is phosphorylated (Anderson et al 2000; Fig. 1). When probing hippocampal homogenate in a Western blot, each antibody recognizes a band at $\sim 72 \mathrm{kD}$, which is the molecular mass of the $\mathrm{Kv} 4.2$ alpha subunit. It is also reassuring that although each antiserum was independently generated against a unique portion of the $\mathrm{Kv} 4.2$ sequence, all three antisera recognize a protein band of identical molecular mass. Our data are indicative of a basal level of phosphorylation of Kv4.2 at the PKA and ERK sites. When each antibody is preincubated with its appropriate antigen, this immunoreactivity disappears (Fig. 2).

\section{Kv4.2 Phospho-Isoforms in the Mouse Brain}

In our initial studies, we examined the distribution of the three phospho-isoforms of Kv4.2 in the mouse brain. Several brain areas manifest immunoreactivity to each antibody including the hippocampus, thalamus, medial habenular nucleus, striatum, amygdala, cortex, and cerebellum, and this immunoreactivity is blocked by preincubation with the appropriate phospho-peptide antigen (Fig. 3). Immunoreactivity is constrained to the soma and dendrites of expressing neurons. This pattern of immunoreactivity is consistent with both the total Kv4.2 protein distribution and Kv4.2 mRNA in brain (Fig. 3), (Sheng et al 1992; Serodio and Rudy 1998). Furthermore, the pattern of ERK triply phosphorylated Kv4.2 immunoreactivity coincides substantially with immunoreactivity seen for phospho-ERK, including overlap

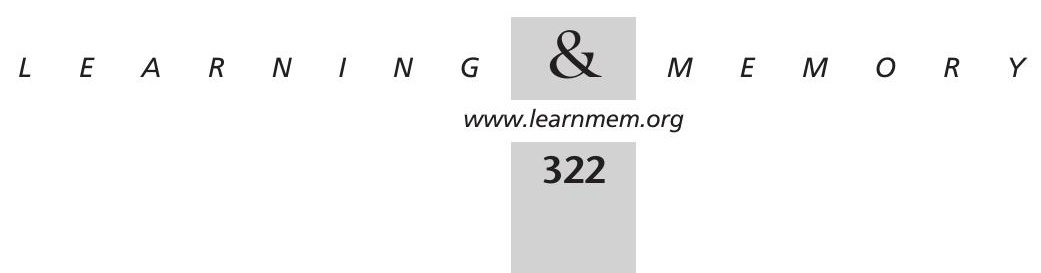




\section{PHOSPHORYLATION SITES ON KV4.2}

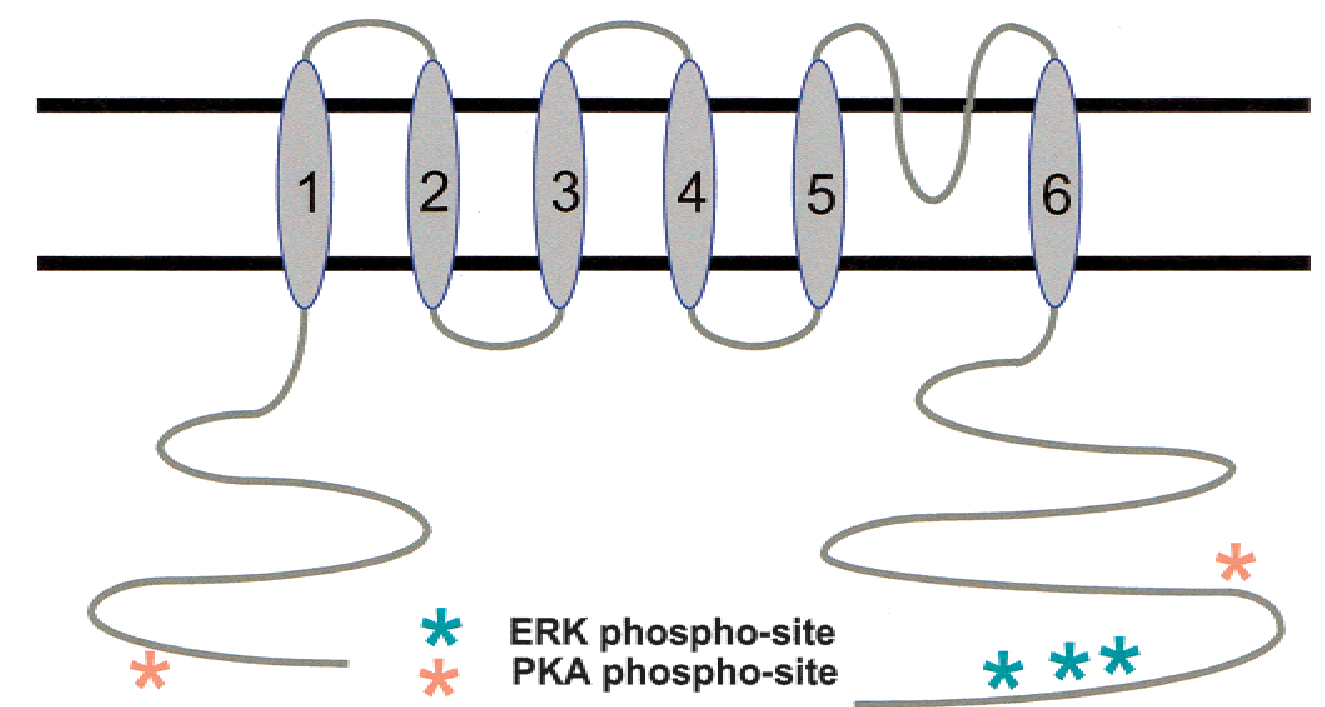

ERK triply phosphorylated Kv4.2 antigen: ${ }_{586}$ LNCEQPYVTTAIISIPTPPVTTPEGDDRPESPE $_{618}$ C-terminal PKA phosphorylated Kv4.2 antigen: ${ }_{546}$ SGSHRGSVQELST ${ }_{558}$ N-terminal PKA phosphorylated Kv4.2 antigen: ${ }_{32}$ RQERKRTQDALIV ${ }_{44}$

Figure 1 Kv4.2 structure and peptide antigens. Schematic structure of Kv4.2 with highlighted ERK and PKA phosphorylation sites. The ERK triply phosphorylated antibody was made against a peptide running from AA586 to AA618, which includes the ERK phosphorylation sites $\mathrm{Thr}^{602}$, $\mathrm{Thr}^{607}$, and Ser ${ }^{616}$. The carboxy-terminal PKA-phosphorylated Kv4.2 antibody was made against a peptide running from AA546 to AA548, which includes a PKA phosphorylation site at $\operatorname{Ser}^{552}$. The amino-terminal PKA-phosphorylated Kv4.2 antibody was made against a peptide running from AA32 to AA44, which includes a PKA phosphorylation site at $\mathrm{Thr}^{38}$.

in the hippocampus, amygdala, thalamus, cortex, and striatum. Our results demonstrate that our phospho-specific antibodies are suitable for localization of Kv4.2 phospho-isoforms using immunohistochemical techniques. Interestingly, they also indicate a prominent presence of phosphorylated Kv4.2 in brain areas known to be involved in associative and motor learning.

\section{Distribution in the Hippocampus}

Kv4.2 is particularly abundant in the hippocampus (Sheng et al 1992), and A-type current density, which is thought to be mediated by $\mathrm{Kv} 4.2$, increases along hippocampal dendrites with distance from the soma (Hoffman et al. 1997). Furthermore, this A-type current can be modulated by PKA activation (Hoffman and Johnston 1998), and Kv4.2 acts as a substrate for both PKA and ERK (Anderson et al. 2000; Adams et al. 2000). Consequently, we were greatly interested in determining the distribution of the basal phosphorylation states of Kv4.2 in the hippocampus by use of our phospho-specific antibodies in immunohistochemistry studies in this area. In CA1, immunoreactivity to the ERK triply phosphorylated $\mathrm{Kv} 4.2$ antibody is predominant in the stratum oriens and stratum radiatum, however, it is minimal in stratum pyramidali (Fig. 4A and Fig. 6A, below; Table 1). In $\mathrm{CA} 3$, the immunoreactivity to the cell soma is striking (Fig 5A). The staining is also strong in the CA3 basilar dendrites in stratum oriens. Staining is conspicuously minimal in the stratum lucidum, which contains CA3 apical dendrites that receive the mossy fiber inputs from dentate granule cells (Fig. 5D). Staining is low but present in the CA1 stratum lacunosum moleculare, which contains CA1 apical dendrites receiving inputs from the entorhinal cortex (Fig. 6A). The molecular layer of the dentate gyrus as well as the granule layer of the dentate gyrus display little immunoreactivity above background. This immunoreactivity in the hippocampus corresponds in general with immunoreactivity to basal phospho-ERK that is present throughout the stratum oriens and stratum radiatum in both CA1 and CA3 (Fig. 4D). Additionally, this immunoreactivity corresponds to the pattern of total $\mathrm{Kv} 4.2$ immunoreactivity with the exception that the CA3 soma stain relatively lightly for total Kv4.2 (Fig. 4E; Sheng et al. 1992).

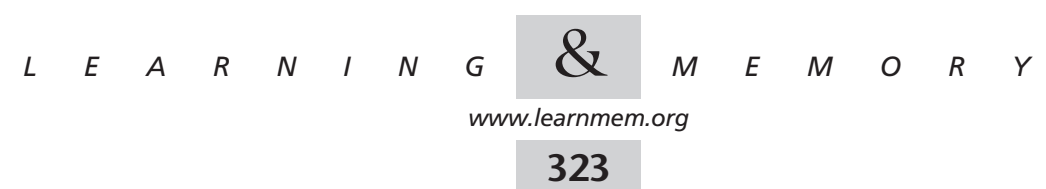


A

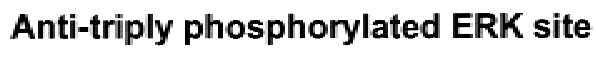

B
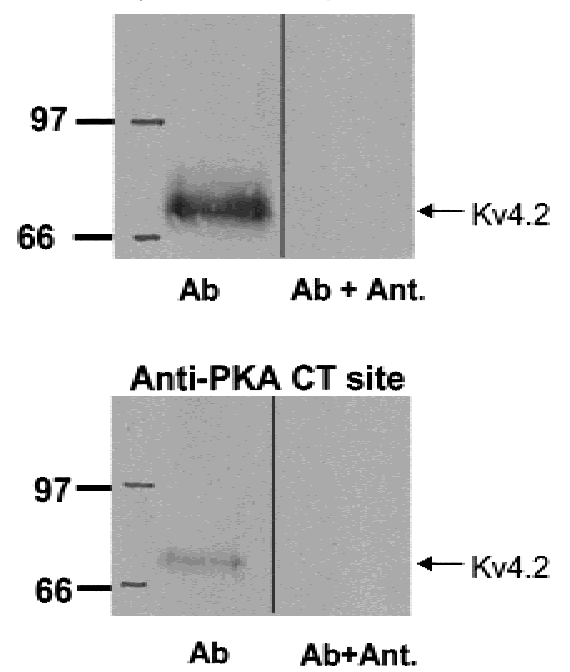

C

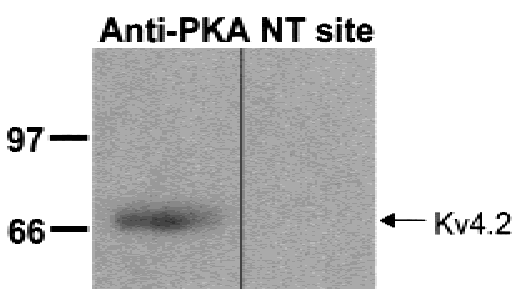

Ab Ab+Ant.

Figure 2 Antibody specificity. Western blotting was performed on hippocampal homogenate membrane preps by use of antibodies against ERK triply phosphorylated Kv4.2 (A), carboxy-terminal PKA-phosphorylated Kv4.2 (B), and amino-terminanl PKA-phosphorylated Kv4.2 (C). Preincubation with the corresponding antigen blocked $90 \%-100 \%$ of immunoreactivity. In $C$, the hippocampus was first treated with forskolin to improve the immunoreactivity.

The pattern of immunoreactivity in the hippocampus with the carboxy-terminal PKA-phosphorylated Kv4.2 antibody is similar to that of the ERK triply phosphorylated Kv4.2 antibody with a few key exceptions. First, there is no staining in the CA3 soma, whereas staining is strong in the CA3 soma with the ERK triply phosphorylated Kv4.2 antibody (Fig. 5A,B). Second, the carboxy-terminal PKA-phosphorylated Kv4.2 antibody shows immunoreactivity to the mossy fiber inputs of stratum lucidum, whereas the ERK triply phosphorylated $\mathrm{Kv} 4.2$ antibody detects nothing there (Fig. 5D,E). Finally, relatively speaking, there is increased staining in the molecular layer of the dentate gyrus with the carboxy-terminal PKA-phosphorylated as compared with the ERK triply phosphorylated Kv4.2 antibody (Fig. 6A,B).

The amino-terminal PKA phosphorylated Kv4.2 antibody exhibits a pattern of immunoreactivity much different from that of the two previous antibodies. In CA1, it is nearly a negative image of its counterparts with little immunore-
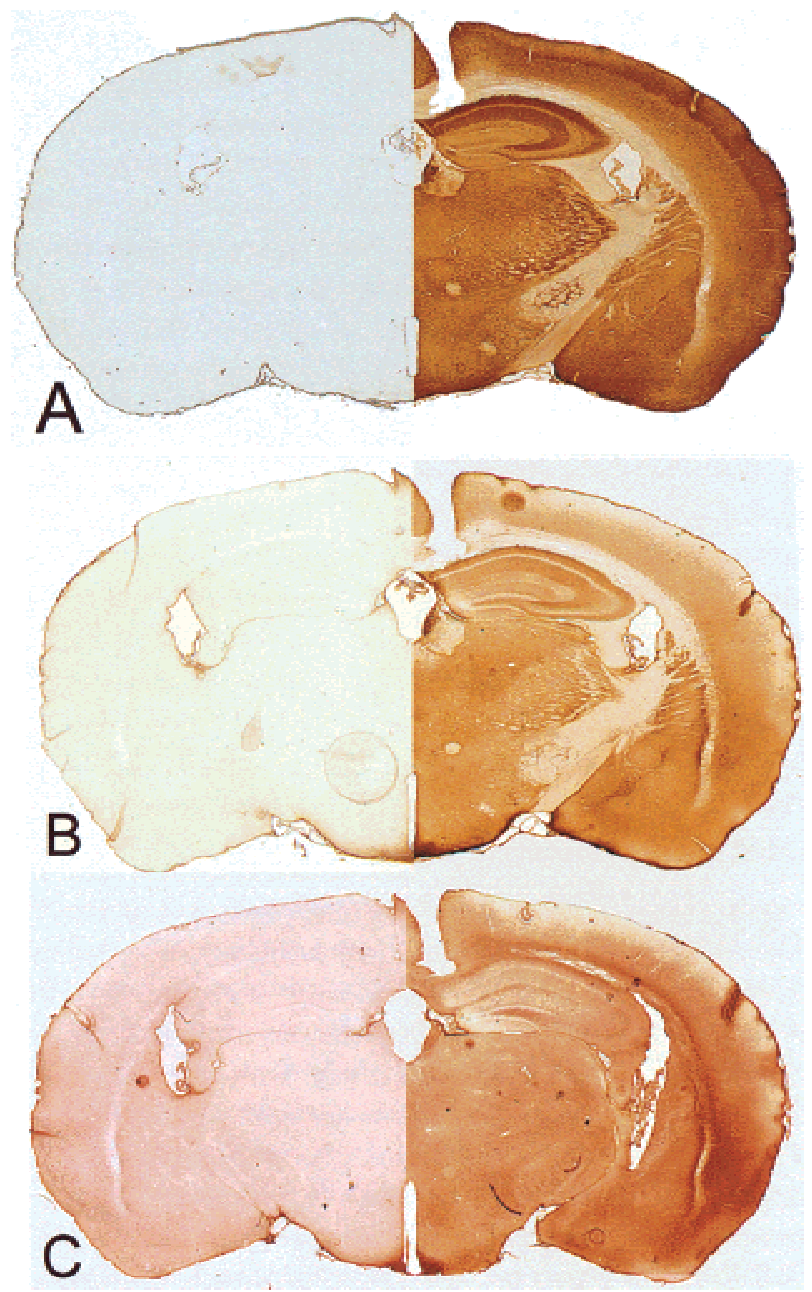

Figure 3 Mouse brain coronal sections. Staining for ERK triply phosphorylated Kv4.2 (A), carboxy-terminal PKA-phosphorylated Kv4.2 (B) and amino-terminal PKA-phosphorylated Kv4.2 (C). The right half in each brain displays immunoreactivity to the appropriate antibody, whereas the left half is representative of the immunoreactivity seen when the antibody is preincubated with its appropriate antigen. In general, staining across antibodies is strong in areas consistent with those described by the pattern of total Kv4.2 staining. Strong immunoreactivity is seen in the hippocampus, thalamus, medial habenular nucleus, striatum, amygdala, cortex, and cerebellum.

activity in stratum oriens or stratum radiatum. Its strongest immunoreactivity is in the stratum lacunosum moleculare (Fig. 6C). There is a low level of immunoreactivity in the CA3 soma (Fig. 5C), but it is far less than the ERK triply phosphorylated Kv4.2 pattern. Like the other two antibodies, there is little immunoreactivity in the molecular layer of the dentate gyrus (Figs. 4C and 6C).

The hippocampus is of particular interest to us given its documented role in both associative and spatial memory. Our findings suggest that the basal phosphorylation state of Kv4.2 within the hippocampus may be specific for the in-

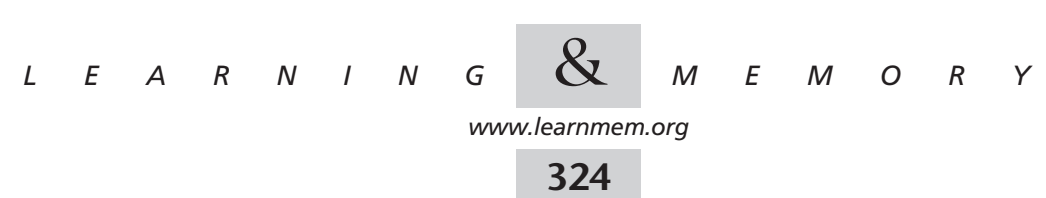




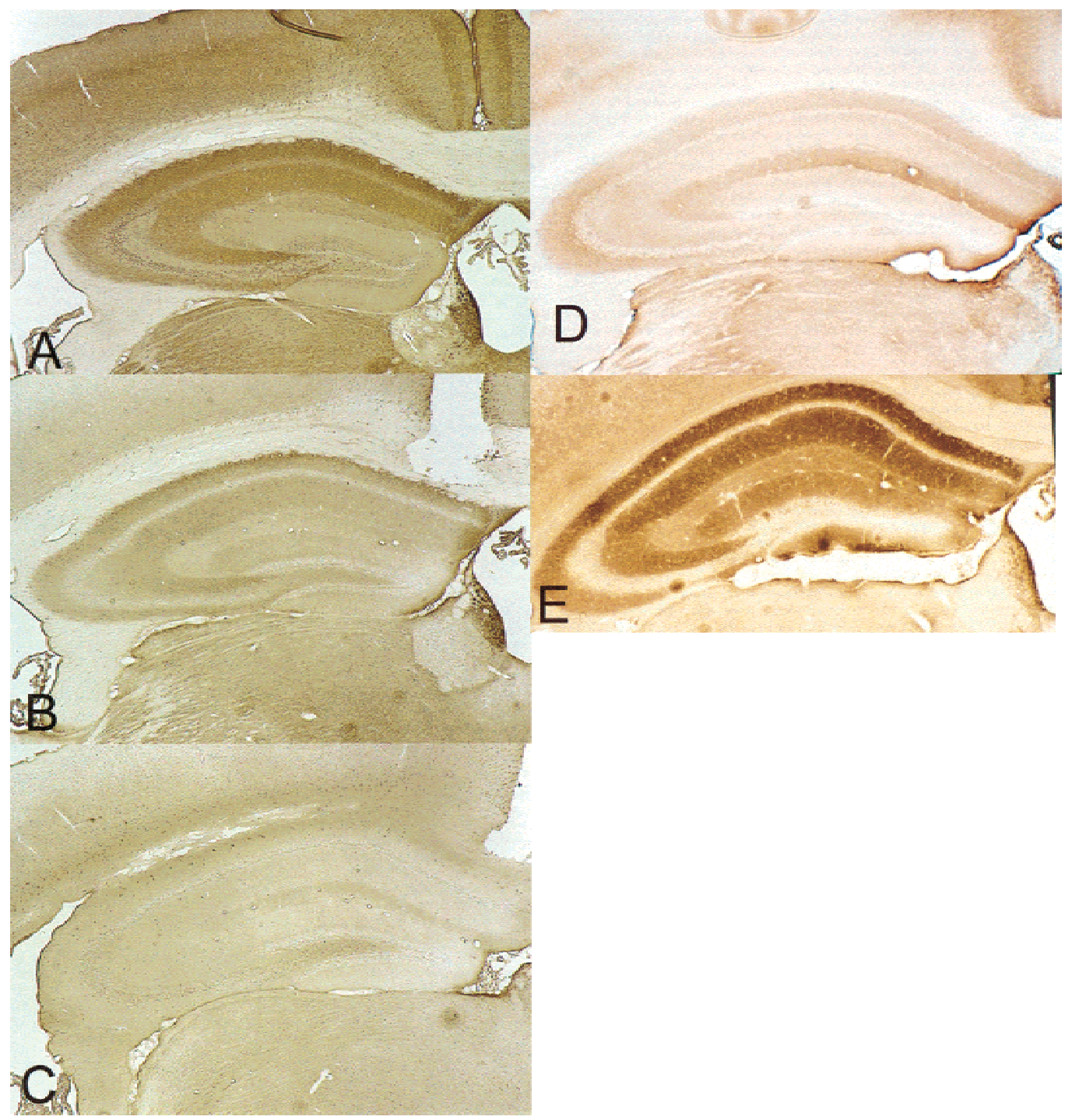

Figure 4 Hippocampus. Staining of ERK triply phosphorylated Kv4.2 (A), carboxy-terminal PKA-phosphorylated Kv4.2 (B), amino-terminal PKA-phosphorylated Kv4.2 (C), phospho-ERK $(D)$, and total Kv4.2 (E) in the hippocampus at 40x. In CA1, immunoreactivity to the ERK triply phosphorylated Kv4.2 antibody is predominant in the stratum oriens and stratum radiatum, however, it is minimal in stratum pyramidali. In CA3, the immunoreactivity to the cell soma is striking. The staining is also strong in the CA3 basilar dendrites in stratum oriens. Staining is conspicuously absent in the stratum lucidum. Staining is low but present in the stratum lacunosum moleculare. The molecular layer of the dentate gyrus as well as the granular layer of the dentate gyrus display little immunoreactivity above background. Areas of overlap with the phospho-ERK antibody $(D)$ include the CA1 stratum oriens and stratum radiatum and the CA3 stratum oriens. Key differences between carboxy-terminal PKA-phosphorylated Kv4.2 immunoreactivity and ERK triply phosphorylated include no staining in the CA3 soma and a greater level of immunoreactivity to the mossy fiber inputs of stratum lucidum $(A, B)$. The amino-terminal PKA-phosphorylated Kv4.2 antibody exhibits a pattern that is nearly a negative image of its counterparts with little immunoreactivity in stratum oriens or stratum readiatum. Its strongest immunoreactivity is in the stratum lacunosum moleculare. There's a small but detectable level of immunoreactivity in the CA3 soma. Like the other two antibodies, there is little immunoreactivity in the dentate gyrus $(C)$.

puts that it receives. An interesting possibility is that phosphorylation of Kv4.2 may be a mechanism for properly targeting $\mathrm{Kv} 4.2$ to specific areas within the hippocampal neuronal soma and dendrites.

\section{Distribution in the Amygdala}

The amygdala is a key structure involved in both cued and contextual learning during fear conditioning. As the biochemical bases for amygdala-dependent learning have been studied in increasing detail, it has been shown that both PKA and ERK play important roles. PKA has been shown to be necessary for LTP in the pathway from external capsule to the lateral nucleus of the amygdala (Huang and Kandel 1998), and ERK and PKA have been shown to be necessary

$$
\begin{array}{lllllllllllllll}
L & E & A & R & N & I & N & G & \mathcal{Q} & M & E & M & O & R & Y \\
\text { www.learnmem.org } & &
\end{array}
$$


Table 1. Summary of Immunoreactivity in the Hippocampus Proper (CA1 and CA3) and the Dentate Gyrus (DG)

\begin{tabular}{|c|c|c|c|c|c|c|c|c|c|c|}
\hline \multirow[b]{2}{*}{ Kinase } & \multicolumn{4}{|c|}{ CA1 } & \multicolumn{3}{|c|}{ CA3 } & \multicolumn{3}{|c|}{ DG } \\
\hline & so & sp & $\mathrm{sr}$ & slm & so & sp & $\mathrm{sl}$ & $\mathrm{ml}$ & $\mathrm{gl}$ & $\mathrm{h}$ \\
\hline ERK & + & - & + & - & + & + & - & - & - & - \\
\hline C-term PKA & + & - & + & - & + & - & + & + & - & \pm \\
\hline N-term PKA & - & - & - & + & - & \pm & - & - & - & + \\
\hline
\end{tabular}

(+) Immunoreactivity is prominent and readily visible in this area; $( \pm)$ immunoreactivity is moderately detectable in this area; (-) immunoreactivity is minimal in this area; (so) stratum oriens; (sp) stratum pyramidali; (sr) stratum radiatum; $(\mathrm{s} / \mathrm{m})$ stratum lacunosum molecular; (ml) molecular layer; (gl) granule cell layer; (h) hilus.

for amygdala LTP (Schafe et 1999). ERK is important in the context of fear conditioning in that it becomes activated in the soma of neurons in the lateral amygdala specifically at 60 min following fear conditioning (G.E. Schafe et al. 2000). It was with this information in mind that we took a closer look at the distribution of the PKA and ERK-phosphorylated isoforms of $\mathrm{Kv} 4.2$ in the amygdala. Immunoreactivity is seen in the soma and processes of neurons in the lateral nucleus, basolateral nucleus, and central nucleus with the ERK triply phosphorylated Kv4.2 antibody (Fig. 7A; Table 2). The same general pattern, although with lesser intensity, is seen with the amino-terminal PKA-phosphorylated Kv4.2 antibody (Fig. 7C). The immunoreactivity to the carboxy-terminal PKA-phosphorylated Kv4.2 antibody is somewhat different. Largely, it seems that only processes are stained and there is much reduced intensity to the central and basolateral nuclei (Fig. 7B).

Although ERK and PKA in the lateral amygdala have been implicated in both LTP and Pavlovian fear-conditioning consolidation, the targets for these kinases are not fully established. One suggested target is CREB, given that ERK has been shown to couple PKA to CREB (Roberson et al.1999) and that CRE- mediated gene transcription increases in the amygdala following fear conditioning (Impey et al. 1998b). Our results are consistent with the idea that Kv4.2 may be another target for ERK and PKA in the amygdala. Furthermore, our results suggest that, as in the hippocampus, Kv4.2 compartmentalization within amygdala neurons, insomuch as relates to soma and/or dendrite expression, may be governed by distinct phosphorylation states.

\section{Distribution in Somatosensory Cortex}

The somatosensory cortex is a site for experience-dependent plasticity. The barrel fields within this area, which are representations of the whisker fields in rodents, are particularly malleable, especially during the critical period. ERK triply phosphorylated Kv4.2 antibodies show predominant staining in layer IV of the somatosensory cortex with pos- sible evidence of barrel staining (Fig. 8A; Table 2). Carboxyterminal PKA-phosphorylated Kv4.2 staining shows weak immunoreactivity in layers V and VI (Fig. 8B). Amino-terminal PKA-phosphorylated Kv4.2 staining displays immunoreactivity in layers IV, V, and VI with the strongest signal in layers V and VI (Fig. 8C). Thus, like the hippocampus, the basal phosphorylation state of Kv4.2 within the somatosensory cortex appears to be correlated with the inputs that it receives. Layer IV receives the inputs mainly from the thalamus and is highlighted by Kv4.2 phosphorylated by ERK. Layers V and VI, which mostly receive intra-cortical inputs, display a basal phosphorylation state of Kv4.2 that seems mostly mediated at Kv4.2's amino terminus by PKA.

\section{Distribution in the Cerebellum}

Given the cerebellum's role in motor learning and Kv4.2's role in regulating generation in cerebellar granule cells (Shibata et al. 2000), we were interested in looking at the Kv4.2 phospho-isoform distribution in the cerebellum. The ERK triply phosphorylated Kv4.2 and the carboxy-terminal PKAphosphorylated Kv4.2 display similar distributions in the cerebellum. Both manifest immunoreactivity in the cerebellar granular layers with no immunoreactivity seen in the Purkinje cell bodies and only modest immunoreactivity in dendrites (Fig. 8D,E; Table 2). Within the granular layer, immunoreactivity is concentrated in numerous small bundles called cerebellar glomeruli. This agrees with previous studies that have described an absence of total Kv 4.2 protein in the molecular layer and glomerular foci in the granular layer (Sheng et al. 1992), as well as an absence of Kv4.2 mRNA in Purkinje cell bodies via in situ hybridization (Serodio and Rudy 1998).

Immunoreactivity to the amino-terminal PKA-phosphorylated Kv4.2 is also absent from Purkinje cell bodies, and contrasting with the other two phospho-isoforms of $\mathrm{Kv} 4.2$, there is only minor immunoreactivity in the granular cell layer (Fig. 8F). There appears to be immunoreactivity in the cerebellar molecular layer that is blocked by preincubation with the appropriate antigen (data not shown). This fact contrasts with a prior report of an absence of Kv4.2 in the cerebellar molecular layer, however, the previous studies detailing the localization of total Kv4.2 protein and mRNA were done in the rat, whereas our studies were done in the mouse. Thus, it is possible that there may be species differences in the localization of Kv4.2 in the cerebellum. The possibility also exists that a minor fraction of total Kv4.2 protein exists in the cerebellar molecular layer, which is highly phosphorylated by PKA at the amino terminus and therefore recognized by our antibody.

\section{DISCUSSION}

The goal of this study was to determine the localization of PKA and ERK-phosphorylated isoforms of the voltage-gated potassium channel Kv4.2, with particular interest in the

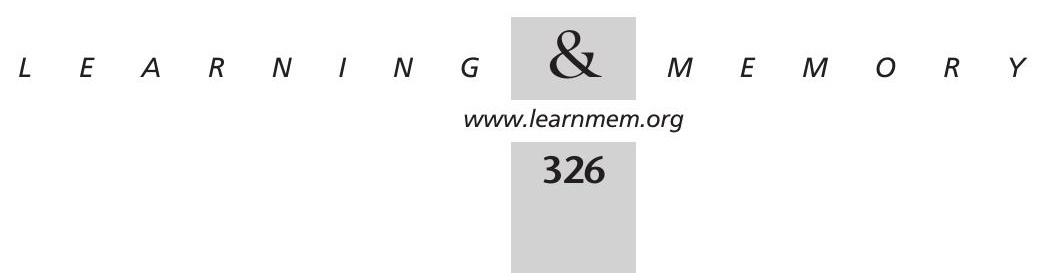




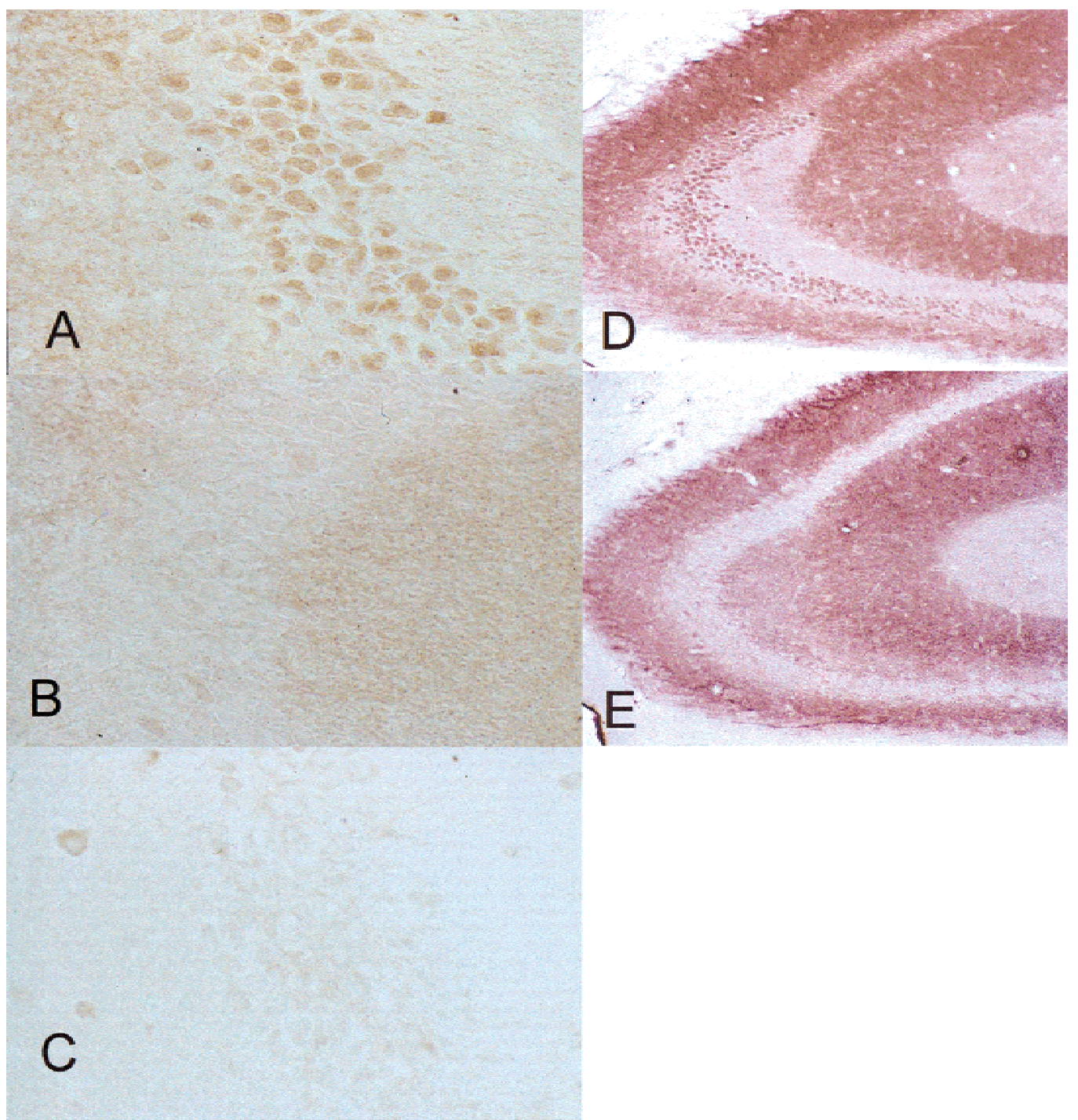

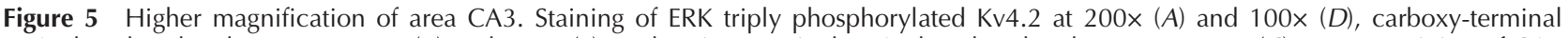

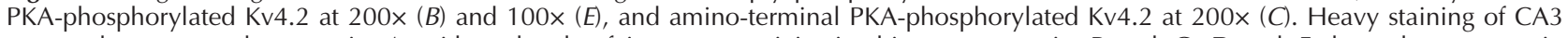
neuronal soma can be seen in $A$, with a dearth of immunoreactivity in this same area in $B$ and $C$. $D$ and $E$ show the contrast in immunoreactivity in the stratum lucidum between the ERK triply phosphorylated Kv4.2 antibody (D) and the carboxy-terminal PKAphosphorylated Kv4.2 antibody (E).

hippocampus. We found that the distribution of these phospho-isoforms of Kv4.2 encompasses a subset of the brain areas highlighted by total Kv4.2, with a differential pattern seen for each phospho-isoform. However, a general trend across all phospho-isoforms was an abundance of immunoreactivity in the hippocampus, amygdala, cortex, and cerebellum, suggesting that ERK and PKA regulation of Kv 4.2 occurs over a broad range of brain areas, particularly areas that have been associated previously with different forms of learning and synaptic plasticity. In its technical aspect, this study demonstrates that the antibodies raised against an ERK triply phosphorylated Kv4.2, a carboxy-terminal PKAphosphorylated Kv4.2, and an amino-terminal PKA-phos- phorylated $\mathrm{Kv} 4.2$ are suitable for immunohistochemistry and reconfirms the observation that $\mathrm{Kv} 4.2$ is a substrate for PKA and ERK in vivo. Furthermore, our observations indicate that these antibodies will be useful for tracking any changes that may occur in the Kv4.2 phosphorylation state in the brain. Changes in the phosphorylation state of both kinases and ion channels are thought to occur during synaptic plasticity and learning; therefore, these phospho-specific antibodies may be particularly useful in detecting any changes that occur during such processes.

The fact that the distribution of a single protein, $\mathrm{Kv} 4.2$, shows a phospho-isoform-specific dependence within a given brain substructure has several interesting implica-

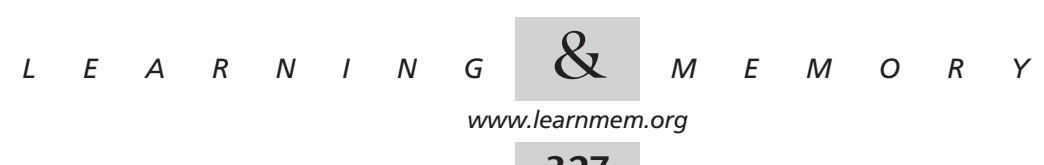




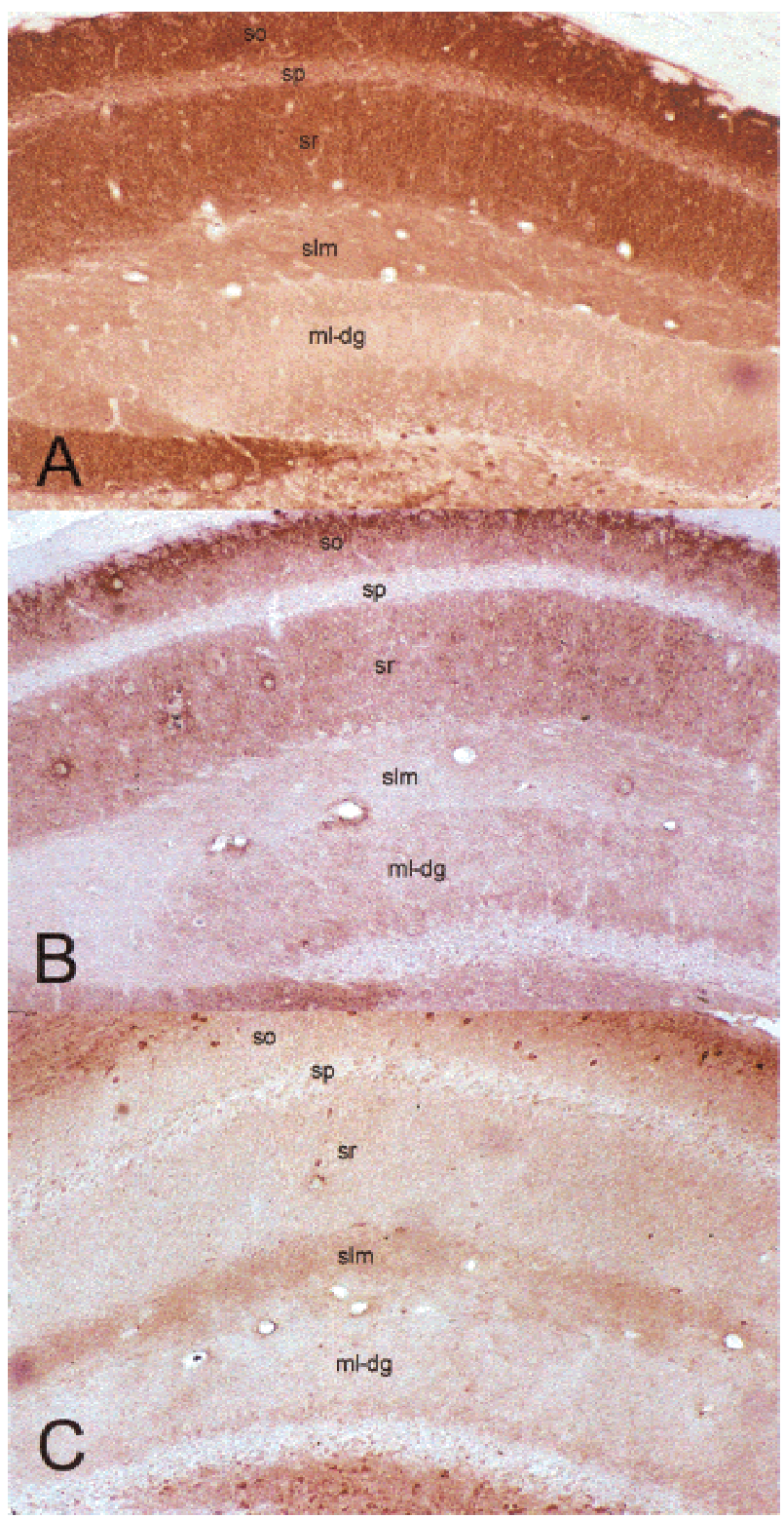

Figure 6 Higher magnification of area CA1. Staining of ERK triply phosphorylated Kv4.2 at $100 \times(A)$, carboxy-terminal PKA-phosphorylated Kv4.2 at $100 \times(B)$, and amino-terminal PKA-phosphorylated Kv4.2 at $100 \times(C)$. Strong immunoreactivity in the CA1 stratum oriens (so) and stratum radiatum (sr) can be seen in $A$ and $B$ with a dearth of immunoreactivity in these same layers in $C$. However, there is a relative dearth of immunoreactivity in the stratum lacunosum moleculare (s $\mathrm{lm}$ ) in $A$ and $B$, whereas immunoreactivity is strong in the slm in $C$. The immunoreactivity in the molecular layer of the dentate gyrus ( $\mathrm{ml}-\mathrm{dg}$ ) is increased in $B$ as compared with $A$ and $C$. (sp) Stratum pyramidali.

tions. One intriguing implication of this differential pattern may be that targeting of $\mathrm{Kv} 4.2$ within these structures is dependent on its phosphorylation state. It has been shown recently that in the hippocampus, the targeting of another voltage-gated potassium channel, Kv2.1, is dependent on a 26-amino acid targeting signal (Lim et al. 2000). Within this signal, four residues were shown to be critical for proper localization via alanine-scanning mutagenesis. Interestingly, three of the four critical amino acids were serines and the fourth was a phenylalanine adjacent to a serine, perhaps implicating phosphorylation at those serines in proper Kv2.1 localization. Because the hippocampus shows such a strong contrast of immunoreactivity between phospho-isoforms of Kv4.2 and because the circuitry of the hippocampus is generally well characterized, it is perhaps the best brain substructure within which to make speculations as to the role of specific phosphorylation in localization. For example, it could be that ERK phosphorylation of Kv4.2 helps to confine $\mathrm{Kv} 4.2$ to the cell soma and basilar dendrites in CA3 and restrict Kv4.2 entry into the apical dendrites that receive mossy fiber inputs, as immunoreactivity is strong in CA3 stratum pyramidali and stratum oriens, but absent in stratum lucidum. Similarly, it may be that amino-terminal PKA phosphorylation of Kv4.2 acts as a molecular visa for entry into the stratum lacunosum moleculare, as immunoreactivity in this area is strongest with the amino-terminal PKA-phosphorylated $\mathrm{Kv} 4.2$, but is relatively weak in the ERK and carboxy-terminal PKA-phosphorylated isoforms of Kv4.2. Future experiments might address the role of phosphorylation in localization within the single neuron by looking at Kv4.2 distribution in cultured hippocampal neurons transfected with Kv4.2 in which the ERK and PKA phosphorylation sites have been eliminated.

Another speculation made on the basis of the differential pattern of Kv4.2 phospho-isoform distribution is that particular molecular mechanisms might exist for different inputs onto particular brain substructures. Again, the hippocampus gives us the clearest example of how this might be the case. For instance, it may be that because $\mathrm{Kv} 4.2$ in the stratum lacunosum moleculare appears to be primarily phosphorylated by PKA at the amino terminus, plasticity of the entorhinal cortex inputs onto distal dendrites of CA1 within the stratum lacunosum moleculare may be principally mediated through PKA. Because Kv4.2 has been shown to be important for regulating membrane excitability and shaping action potentials, phosphorylation of Kv4.2 at discrete junctions could affect the overall output of the hippocampal circuit. Although the laminations in the cortex are not as striking as in the hippocampus, a similar argument might be derived on the basis of the pattern of immunoreactivity seen there. For example, a strong level of immunoreactivity to the ERK-phosphorylated Kv4.2 is observed in layer IV of the cortex, a layer that primarily receives afferents from the thalamus. Layers $\mathrm{V}$ and VI of the cortex show predominant immunoreactivity to the aminoterminal PKA-phosphorylated antibody. Thus, one may infer that plasticity of the thalamo-cortical inputs onto layer IV may be mediated more by ERK, whereas plasticity of cor-

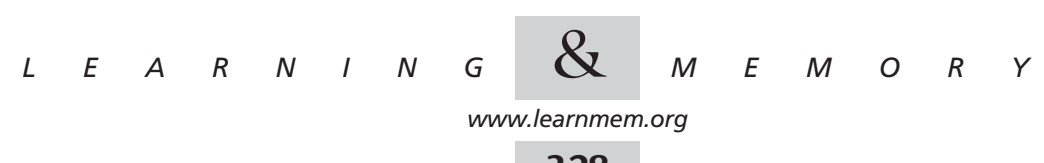




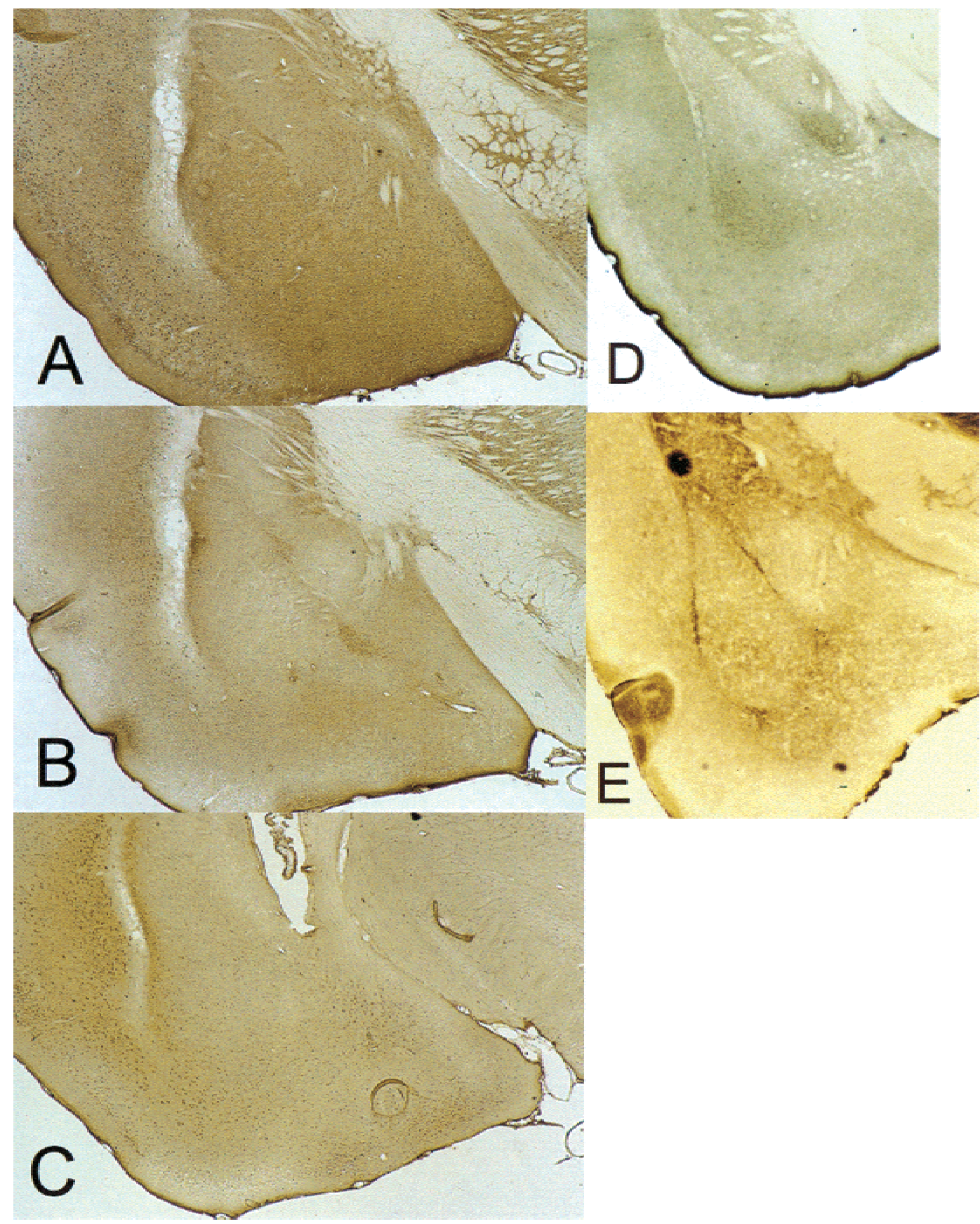

Figure 7 Amygdala. Staining of ERK triply phosphorylated Kv4.2 (A), carboxy-terminal PKA-phosphorylated Kv4.2 (B) and amino-inal PKA-phosphorylated Kv4.2 (C), phospho-ERK (D), and total Kv4.2 (E) in the amygdala at $40 \times$. Immunoreactivity is seen in the soma and processes of neurons in the lateral nucleus, basolateral nucleus, and central nucleus with the ERK triply phosphorylated Kv4.2 antibody (A). The same pattern, although with lesser intensity, is seen with the amino-terminal PKA-phosphorylated Kv4.2 antibody (C). The immunoreactivity to the carboxy-terminal PKA-phosphorylated Kv4.2 antibody is somewhat different. It seems that only processes are stained and there is much reduced intensity to the central and basolateral nuclei $(B)$.

tico-cortical inputs onto layers $\mathrm{V}$ and VI may be mediated more by PKA. A corollary of these considerations is that input specificity of molecular mechanisms for plasticity appears to cross brain substructure boundaries.
One major caveat of our studies is that the functional effect of Kv4.2 phosphorylation by ERK and PKA remains largely unknown. Future studies detailing the ways in which ERK and PKA influence Kv4.2 channel biophysical proper-

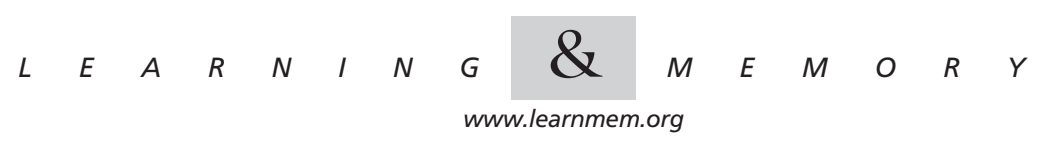


Table 2. Summary of Immunoreactivity in the Amygdala, Cortex, and Cerebellum

\begin{tabular}{|c|c|c|c|}
\hline Kinase & Amygdala & Cortex & Cerebellum \\
\hline ERK & $\begin{array}{l}\text { Soma and } \\
\text { processes of LA, } \\
\text { BLA, and CE }\end{array}$ & Layer IV & $\begin{array}{c}\text { Granular } \\
\text { layer }\end{array}$ \\
\hline $\begin{array}{l}\text { C-term } \\
\text { PKA }\end{array}$ & Processes of LA & $\begin{array}{l}\text { Layers } \mathrm{V} \\
\text { and } \\
\mathrm{VI}^{\text {(a) }}\end{array}$ & $\begin{array}{c}\text { Granular } \\
\text { layer }\end{array}$ \\
\hline $\begin{array}{c}\text { N-term } \\
\text { PKA }\end{array}$ & $\begin{array}{l}\text { Soma and } \\
\text { processes of LA, } \\
\text { BLA, and CE }\end{array}$ & $\begin{array}{l}\text { Layers IV, } \\
\text { V, and } \\
\text { VI }\end{array}$ & $\begin{array}{l}\text { Molecular } \\
\text { layer }\end{array}$ \\
\hline
\end{tabular}

The sub-areas listed are representative of the region within each given structure displaying the highest level of immunoreactivity. (LA) Lateral nucleus of the amygdala; (BLA) basolateral nucleus of the amygdala; (CE) central nucleus of the amygdala (a) although these layers display the greatest level of immunoreactivity in the cortex, the immunoreactivity is weak in general.

ties will be useful in validating aspects of this hypothesis. Given that the activation curve for hippocampal A-type current shifts in the depolarizing direction after application of PKA and PKC activators (Hoffman and Johnston 1998) and the evidence correlating A-type current to Kv4.2 expression (Shibata et al. 2000; Tkatch et al. 2000;), it seems likely that ERK or PKA phosphorylation may mediate biophysical effects on $\mathrm{Kv} 4.2$ channel function. In this case, the present observations have an interesting implication; phosphorylation-dependent alterations in $\mathrm{Kv} 4.2$ functional properties may serve to fine tune the membrane electrical properties for specific synaptic inputs.

In conclusion, our results indicate that there exists an input-specific distribution of differentially phosphorylated Kv4.2 in the mouse brain with primary immunoreactivity in brain areas associated previously with associative, spatial, and motor learning. These results provide an anatomical context in which to place future neurophysiological and molecular data determining the precise role of ERK and PKA phosphorylation of Kv4.2.

\section{METHODS}

\section{Sample Preparation}

Mouse brains were immediately removed and perfused with icecold saline (125 mM NaCl, $2.5 \mathrm{mM} \mathrm{KCl}, 1.25 \mathrm{mM} \mathrm{NaH}_{2} \mathrm{PO}_{4}, 25 \mathrm{mM}$ $\mathrm{NaHCO}_{3}, 25 \mathrm{mM}$ D-glucose, $2 \mathrm{mM} \mathrm{CaCl}, 1 \mathrm{mM} \mathrm{MgCl}_{2}$, saturated with $95 \% \mathrm{O}_{2} / 5 \% \mathrm{CO}_{2}$ at $\mathrm{pH} 7.4$ ). Hippocampi were dissected, then homogenized in $2.5 \mathrm{ml}$ of homogenization buffer (HB) (20 mM Tris- $\mathrm{HCl}$ at $\mathrm{pH}$ 7.5, $1 \mathrm{mM}$ EGTA, $1 \mathrm{mM}$ EDTA, $25 \mu \mathrm{g} / \mathrm{ml}$ aprotonin, $25 \mu \mathrm{g} / \mathrm{ml}$ leupeptin, $1 \mathrm{mM} \mathrm{Na}{ }_{4} \mathrm{P}_{2} \mathrm{O}_{7}, 500 \mu \mathrm{M}$ PMSF, $4 \mathrm{mM}$ paranitrophenylphosphate, $1 \mathrm{mM}$ sodium orthovanadate). This homogenate was then spun at $3000 \mathrm{RPM}$ at $4^{\circ} \mathrm{C}$ for $2 \mathrm{~min}$ in a tabletop ultracentrifuge. The supernatant was collected and spun at 60,000 RPM $(100,000 \mathrm{~g})$ at $4^{\circ} \mathrm{C}$ for $20 \mathrm{~min}$ in a Beckman ultracentrifuge. The resultant pellet was suspended in $75 \mu \mathrm{L}$ of $10 \%$ SDS containing
$100 \mathrm{mM}$ DTT, $10 \mu \mathrm{g} / \mathrm{mL}$ pepstatin, $10 \mu \mathrm{g} / \mathrm{mL}$ aprotinin, $10 \mu \mathrm{g} / \mathrm{mL}$ leupeptin, $80 \mathrm{ng} / \mathrm{mL}$ microcystin, and $100 \mu \mathrm{M}$ PMSF and $25 \mu \mathrm{L}$ of $4 \mathrm{x}$ sample buffer containing $100 \mathrm{mM}$ DTT

\section{Antibody Development}

The development of the phospho-specific antibodies has been described previously for the ERK triply phosphorylated Kv4.2 antibody (Adams et al. 2000 ) and the carboxy-terminal and aminoterminal PKA-phosphorylated Kv4.2 antibodies (Anderson et al. 2000). Briefly, the phospho-peptide antigens (listed in Fig. 1) were coupled to keyhole limpet hemocyanin and injected into rabbits. Polyclonal antisera were harvested and affinity purified against the appropriate synthetic phospho-peptide using Hi-trap columns (Amersham Pharmacia Biotech).

\section{Western Blotting}

Hippocampal homogenate membrane proteins were loaded and run on a $10 \%$ acrylamide gel. Gels were then blotted electrophoretically to Immobilon filter paper with a transfer tank maintained at $4^{\circ} \mathrm{C}$, with typical parameters being a 1.5 -hr transfer at a constant current of 600 milliamps (transfer buffer: $192 \mathrm{mM}$ glycine, $25 \mathrm{mM}$ Tris at $\mathrm{pH}$ 8.3). Immobilon filters were blocked for $1 \mathrm{hr}$ at room temperature in Blotto $(10 \mathrm{mM}$ Tris- $\mathrm{HCl}$ at $\mathrm{pH} 7.5,150 \mathrm{mM} \mathrm{NaCl}$, $0.05 \%$ Tween $20,5 \%$ powdered milk, and $0.01 \%$ thimerosal). All antibody applications were done in Blotto. Primary antibody concentrations: ERK triply phosphorylated Kv4.2 (1:300), carboxy-terminal PKA-phosphorylated Kv4.2 (1:500), amino-terminal PKA phosphorylated Kv4.2 (1:100).

Antibody detection. Immobilon filters were incubated sequentially with primary antibody and a biotin-labeled goat anti-rabbit IgG secondary antiserum $(1: 20,000)$ and then developed by enhanced chemiluminescence (ECL, Amersham). Blots were washed extensively in TTBS $(50 \mathrm{mM}$ Tris- $\mathrm{HCl}$ at $\mathrm{pH} 7.5,150 \mathrm{mM} \mathrm{NaCl}, 0.05 \%$ Tween 20) after incubations with primary antibody, second antibody, or $\mathrm{ABC}$ reagents (typically four washes, each $10 \mathrm{~min}$ )

\section{Immunohistochemistry}

Adult mice were anesthetized intraperitoneally with ketamine/xylazine and perfused transcardially with $10 \mathrm{~mL}$ of $0.9 \% \mathrm{NaCl}$ followed by $50 \mathrm{~mL}$ of $3 \%$ PFA $1 \%$ gluteraldehyde in PBS (pH 7.4). The brains were then cryoprotected in $30 \%$ sucrose for $24-48 \mathrm{hr}$ at $4^{\circ} \mathrm{C}$ until they sank. Brains were then frozen and mounted for cryostat sectioning. Twenty-micron sections were cut and immediately thawmounted on Plus slides. Sections were incubated in $0.3 \% \mathrm{H}_{2} \mathrm{O}_{2}$ in methanol for $30 \mathrm{~min}$ at room temperature, washed in PBST (PBS containing 0.2\% Triton X-100), and blocked in PBST containing 5\% normal goat serum (NGS). Blocking media was blotted from the slides, and sections were then incubated with an appropriate dilution of primary antibody for $48 \mathrm{hr}$ in a humidified chamber at $4{ }^{\circ} \mathrm{C}$ (anti-ERK triply phosphorylated Kv4.2 (1:100), anti-carboxy-terminal PKA-phosphorylated Kv4.2 (1:100), anti-amino-terminal PKAphosphorylated Kv4.2 (1:10), anti-total Kv4.2 (Chemicon Labs) (1: 100), and anti-phospho-ERK (New England Biolabs) (1:100). Sections were washed again in PBST and incubated with a 1:200 dilution of goat anti-rabbit biotinylated secondary antibody for 30 min at room temperature (Pierce ImmunoPure $A B C$ Peroxidase Rabbit IgG Staining Kit). Sections were washed again with PBST and then incubated with a 1:50 dilution of $\mathrm{ABC}$ reagent (Pierce) for $30 \mathrm{~min}$ at room temperature. Sections were again washed in PBST, and staining was revealed with metal-enhanced DAB (Pierce). Sections were then rinsed, cleared, and coverslipped using a xylene-

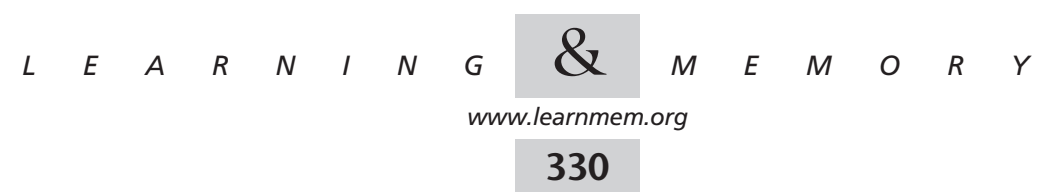




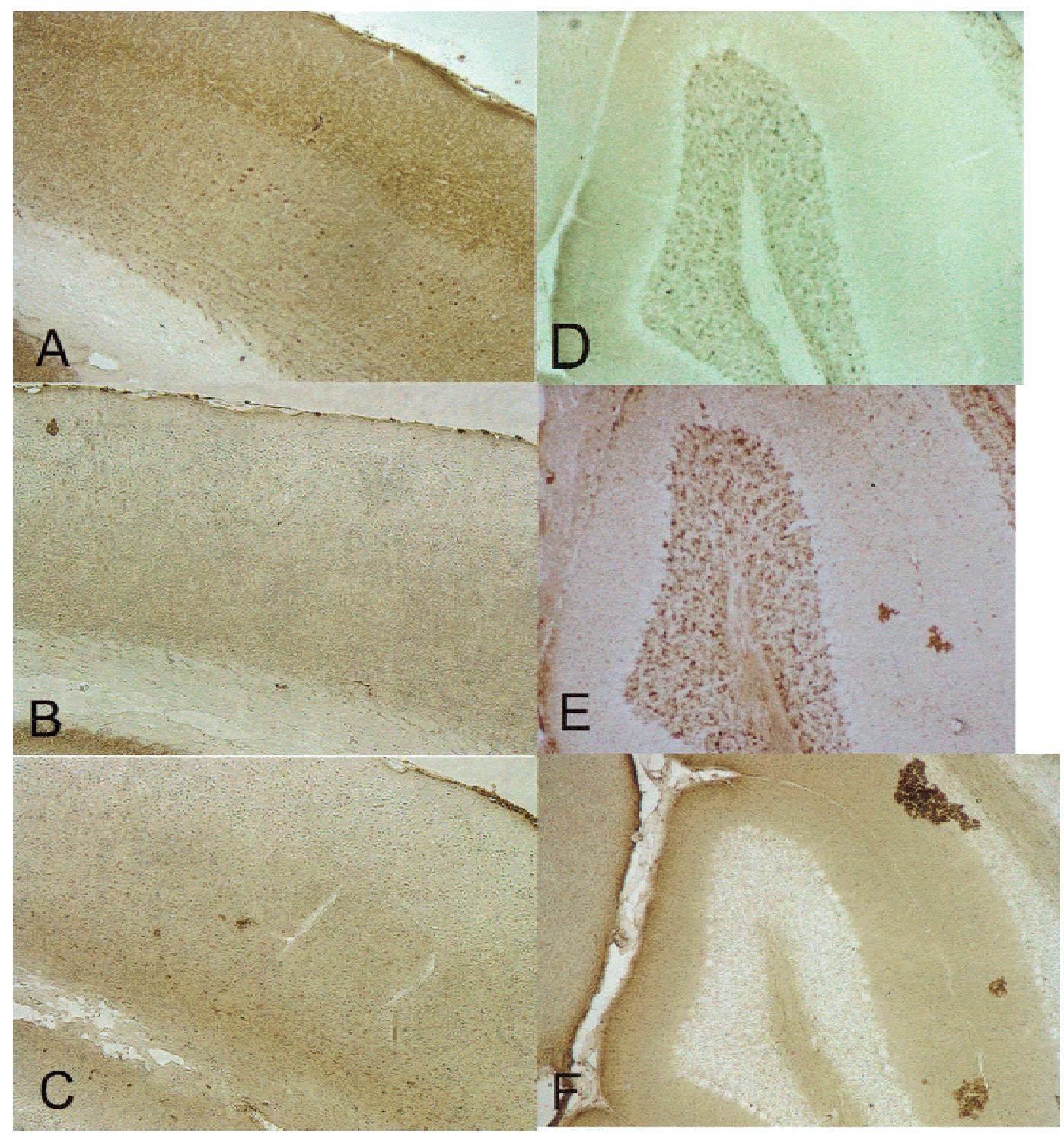

Figure 8 Cortex and Cerebellum. Staining of ERK triply phosphorylated Kv4.2 $(A, D)$, carboxy-terminal PKA-phosphorylated Kv4.2 (B,E), and amino-terminal PKA-phosphorylated Kv4.2 $(C, F)$ in the cortex $(A, B, C)$ and cerebellum $(D, E, F)$ at 100x. In the cortex, ERK triply phosphorylated Kv4.2 antibodies show predominant staining in layer IV of the cortex with possible evidence of barrels (A). Carboxy-terminal PKAphosphorylated Kv4.2 staining shows weak immunoreactivity in layers $\mathrm{V}$ and $\mathrm{VI}(B)$. Amino-terminal PKA-phosphorylated Kv4.2 staining displays immunoreactivity in layers IV, $\mathrm{V}$, and $\mathrm{VI}$ with the strongest signal in layers $\mathrm{V}$ and $\mathrm{VI}(C)$. In the cerebellum, staining with the ERK triply phosphorylated and carboxy-terminal PKA-phosphorylated Kv4.2 is nearly identical. Strong staining can be visualized in the granular layer, however, staining is absent from the Purkinje cell bodies and is only minor in the molecular layer $(D, E)$. In contrast to the other two antibodies, amino-terminal PKA-phosphorylated Kv4.2 antibodies recognize the molecular layer, and staining is highly reduced in the granular layer. Purkinje cell bodies are void of immunoreactivity.

based medium. The images presented are representative of the immunoreactivity observed across multiple brain slices from three or more animals. Staining was graded as high or low by observers who were blind to the particular antibody utilized.

\section{ACKNOWLEDGMENTS}

We thank Angela Major and Ron Broide for technical assistance with the immunohistochemistry. This work was supported by National Institute of Health Grants 37444 and MH 57014 (J.D.S.), NS 01836 (A.E.A.), and a NARSAD Independent Investigator Award (J.D.S.)

The publication costs of this article were defrayed in part by payment of page charges. This article must therefore be hereby marked "advertisement" in accordance with 18 USC section 1734 solely to indicate this fact.

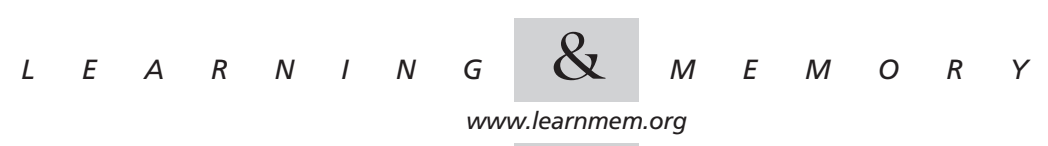




\section{REFERENCES}

Adams, J.P., Anderson, A.E., Varga, A.W., Dineley, K.T., Cook, R.G., Pfaffinger, P.J., and Sweatt, J.D. 2000. The A-type potassium channel $\mathrm{Kv} 4.2$ is a substrate for the nitrogen-activated protein kinase erk. $J$. Neurochem. (in press)

Alonso, G. and Widmer, H. 1997. Clustering of KV4.2 potassium channels in Postsynaptic membrane of rat supraoptic neurons: An ultrastructural study. Neuroscience 77: 617-621.

Anderson, A.E., Adams, J.P., Qian, Y., Cook, R.G., Pfaffinger, P.J., and Sweatt, J.D. 2000. Kv4.2 phosphorylation by cyclic AMP-dependent protein kinase. J. Biol. Chem. 275: 5337-5346.

Atkins, C.M., Selcher, J.C., Petraitis, J.J., Trzaskos, J.M., and Sweatt, J.D. 1998. The ERK cascade is required for mammalian associative learning. Nat. Neurosci. 1: 602-609.

Barry, D.M., Xu, H., Schuessler, R.B., and Nerbonne, J.M. 1998. Functional knockout of the transient outward current, long-QT syndrome, and cardiac remodeling in mice expressing a dominant-negative Kv4 alpha subunit. Circ. Res. 83: 560-567.

Blum, S., Moore, A.N., Adams, F., and Dash, P.K. 1999. A mitogen-activated protein kinase cascade in the CA1/CA2 subfield of the dorsal hippocampus is essential for long-term spatial memory. $J$. Neurosci.. 19: 3535-3544.

English, J.D. and Sweatt, J.D. 1997. A requirement for the mitogen-activated protein kinase cascade in hippocampal long term potentiation. J. Biol. Chem. 272: 19103-19106.

Hoffman, D.A. and Johnston, D. 1998. Downregulation of transient K+ channels indendrites of hippocampal CA1 pyramidal neurons by activation of PKA and PKC. J. Neurosci. 18: 3521-3528.

Hoffman, D.A., Magee, J.C., Colbert, C.M., and Johnston, D. 1997. K+ channel regulation of signal propagation in dendrites of hippocampal pyramidal neurons. Nature 387: 869-875.

Huang, Y.Y. and Kandel, E.R. 1998. Postsynaptic induction and PKA-dependent expression of LTP in the lateral amygdala. Neuron 21: $169-178$.

Impey, S., Obrietan, K., Wong, S.T., Poser, S., Yano, S., Wayman, G., Deloulme, J.C., Chan, G., and Storm, D.R. 1998a. Cross talk between ERK and PKA is required for Ca2+ stimulation of CREB-dependent transcription and ERK nuclear translocation. Neuron 21:: 869-883.

Impey, S., Smith, D.M., Obrietan, K., Donahue, R., Wade, C., and Storm D.R. 1998b. Stimulation of cAMP response element (CRE)-mediated transcription duringcontextual learning. Nat. Neurosci. 1: 595-601.

Lim, S.T., Antonucci, D.E., Scannevin, R.H., and Trimmer, J.S. 2000. A novel targeting signal for proximal clustering of the Kv2.1 $\mathrm{K}+$ channel in hippocampal neurons. Neuron 25: 385-397.

Maletic-Savatic, M., Lenn, N.J., and Trimmer, J.S. 1995. Differential spatiotemporal expression of $\mathrm{K}+$ channel polypeptides in rat hippocampal neurons developing in situ and in vitro. J. Neurosci. 15: 3840-3851.

Roberson, E.D., English, J.D., Adams, J.P., Selcher, J.C., Kondratick, C., and Sweatt, J.D. 1999. The mitogen-activated protein kinase cascade couples PKA and PKC to cAMP response element binding protein phosphorylation in area CA1 of hippocampus. J. Neurosci. 19: 4337-4348.

Schafe, G.E., Nadel, N.V., Sullivan, G.M., Harris, A., and LeDoux, J.E. 1999. Memory consolidation for contextual and auditory fear conditioning is dependent on protein synthesis, PKA, and MAP kinase. Learn. $\&$ Mem.. 6: 97-110.

Schafe, G.E., Atkins, C.M., Swank, M.E., Bauer, E.P., Sweatt, J.D., and LeDoux, J.E. 2000. Activation of ERK/MAP kinase in the amygdala is required for memory consolidation of Pavlovian fear conditioning. $J$. Neurosci. (in press).

Selcher, J.C., Atkins, C.M., Trzaskos, J.M., Paylor, R., and Sweatt, J.D. 1999. A necessity for MAP kinase activation in mammalian spatial learning Learn. \& Mem.. 6: 478-490.

Serodio, P. and Rudy, B. 1998. Differential expression of Kv4 K+ channel subunits mediating subthreshold transient $\mathrm{K}+$ (A-type) currents in rat brain. J. Neurophysiol.. 79: 1081-1091.

Serodio, P., Vega-Saenz de Miera, E., and Rudy, B. 1996. Cloning of a novel component of A-type $\mathrm{K}+$ channels operating at subthreshold potentials with unique expression in heart and brain. J. Neurophysiol.. 75: 2174-2179.

Sheng, M., Tsaur, M.L., Jan, Y.N., and Jan, L.Y. 1992. Subcellular segregation of two A-type $\mathrm{K}+$ channel proteins in rat central neurons. Neuron 2: 271-284.

Shibata, R., Nakahira, K., Shibasaki, K., Wakazono, Y., Imoto, K., and Ikenaka, K. 2000. A-Type K+ current mediated by the Kv4 channel regulates the generation of action potential in developing cerebellar granule cells. J. Neurosci. 20: 4145-4155

Song, W.J., Tkatch, T., Baranauskas, G., Ichinohe, N., Kitai, S.T., and Surmeier, D.J. 1998. Somatodendritic depolarization-activated potassium currents in rat neostriatal cholinergic interneurons are predominantly of the A type and attributable to coexpression of Kv4.2 and Kv4.1 subunits. J. Neurosci.. 18: 3124-3137.

Tkatch, T., Baranauskas, G., and Surmeier, D.J. 2000. Kv4.2 mRNA abundance and A-type $\mathrm{K}(+)$ current amplitude are linearly related in basal ganglia and basal forebrain neurons. J. Neurosci. 20: 579-588.

Wong, S.T., Athos, J., Figueroa, X.A., Pineda, V.V., Schaefer, M.L., Chavkin, C.C., Muglia, L.J., and Storm, D.R. 1999. Calcium-stimulated adenylyl cyclase activity is critical for hippocampus-dependent long-term memory and late phase LTP. Neuron 23: 787-798.

Received June 26, 2000; accepted in revised form July 26, 2000. 


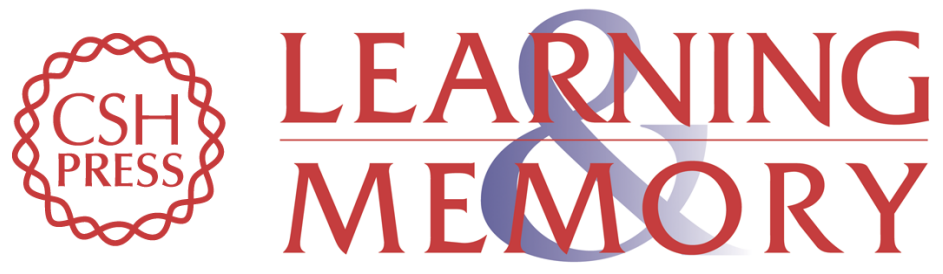

\section{Input-Specific Immunolocalization of Differentially Phosphorylated Kv4.2 in the Mouse Brain}

Andrew W. Varga, Anne E. Anderson, J. Paige Adams, et al.

Learn. Mem. 2000, 7:

Access the most recent version at doi:10.1101/lm.35300

References This article cites 23 articles, 12 of which can be accessed free at: http://learnmem.cshlp.org/content/7/5/321.full.html\#ref-list-1

License

Email Alerting Receive free email alerts when new articles cite this article - sign up in the box at the Service top right corner of the article or click here. 